\title{
Schisandrin B attenuates the inflammatory response, oxidative stress and apoptosis induced by traumatic spinal cord injury via inhibition of $\mathbf{p 5 3}$ signaling in adult rats
}

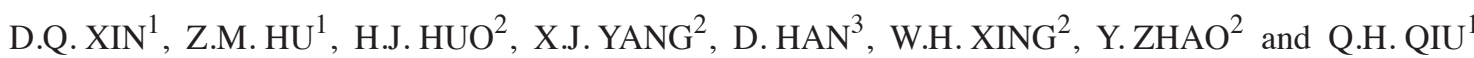 \\ ${ }^{1}$ Department of Orthopedics, The First Affiliated Hospital of Chongqing Medical University, Chongqing \\ 400016; ${ }^{2}$ Department of Spinal Surgery, The Second Affiliated Hospital of Inner Mongolia Medical \\ University, Hohhot, Inner Mongolia 010030; ${ }^{3}$ Department of Cardiovascular Medicine, The Affiliated \\ Hospital of Inner Mongolia Medical University, Hohhot, Inner Mongolia 010059, P.R. China
}

Received February 17, 2016; Accepted February 20, 2017

DOI: $10.3892 / \mathrm{mmr} .2017 .6622$

\begin{abstract}
Schisandrin B is an active monomer of the Chinese magnolia vine (Schisandra chinensis) that can reduce transaminase activity in liver cells, inhibit lipid peroxidation, enhance antioxidant status, has protective effects in the liver and has antitumor effects. The present study investigated the potential protective effects of schisandrin $B$ on the p53 signaling pathway in attenuating the inflammatory response, oxidative stress and apoptosis induced by traumatic spinal cord injury (TSCI) in adult rats. Behavioral examination, inclined plate test and spinal cord water content were used to evaluate the protective effect of schisandrin B in TSCI rats. The expression levels of superoxide dismutase (SOD), malondialdehyde (MDA), nuclear factor (NF)-kB subunit p65 and tumor necrosis factor (TNF)- $\alpha$ were examined using ELISA kits. Western blot analysis was performed to analyze the protein expression of caspase- 3 and phosphorylated (p)-p53 in TSCI rats. In the present study, schisandrin B improved behavioral examination results and the maximum angle of inclined plate test, and inhibited spinal cord water content in rats with TSCI. Notably, schisandrin B reduced the activation of traumatic injury-associated pathways, including SOD, MDA, NF- $\kappa B$ p65 and TNF- $\alpha$, in TSCI rats. In addition, schisandrin $\mathrm{B}$ suppressed the TSCI-induced expression of caspase- 3 and p-p53 in TSCI rats. These results indicated that schisandrin B may attenuate the inflammatory response, oxidative stress and apoptosis in TSCI rats by inhibiting the p53 signaling pathway in adult rats.
\end{abstract}

Correspondence to: Dr Z.M. Hu, Department of Orthopedics, The First Affiliated Hospital of Chongqing Medical University, 1 Yixueyuan Road, Yuzhong, Yuanjia Gang, Chongqing 400016, P.R. China

E-mail: zhenming_hu@163.com

Key words: schisandrin B, traumatic spinal cord injury, inflammatory response, oxidative stress, apoptosis

\section{Introduction}

Spinal cord injury (SCI) is a highly disabling injury; with expensive treatments and other costs, loss of labor and serious complications, $\mathrm{SCI}$ is a heavy burden for the patient's family and society (1). Epidemiological studies on traumatic SCI (TSCI) are regularly conducted worldwide; however, these studies are less common in China (2). The male to female ratio ranges between 2.5:1 and 6:1, and as average life expectancy increases the incidence increases every year in China (3). The mortality rate in patients with TSCI is relatively high; in fact, the United States reported a mortality rate of 25.5-30.0/106 with the cause of mortality most commonly associated with diseases in the circulatory and respiratory systems (3). In addition, TSCI is associated with a number of severe complications, including infection, bedsores and deep vein thrombosis (3). Owing to these severe complications, it is important to investigate effective treatment methods to constantly monitor TSCI progress (4). The underlying mechanisms of TSCI-associated damage are beginning to be revealed; however, identifying effective treatments for TSCI has remained challenging (4). A number of different drug-based treatments, surgical treatments and physical rehabilitation methods have had some success; however, they do have some limitations (4).

Schisandrin B and schizandrin are the main active ingredients in the fruit of the Chinese magnolia vine (Schisandra chinensis). Previous in vivo and in vitro studies have demonstrated that schisandrin B scavenges free radicals and inhibits lipid peroxidation (5). Schisandrin B has a high level of activity in the central nervous system, the cardiovascular system and the respiratory systems, and it has a regulatory role in the stimulation of the cerebral cortex inhibition process $(5,6)$. The aim of present study was to evaluate the effects of schisandrin B on TSCI, and to verify whether schisandrin B markedly inhibits the expression of pro-inflammatory factors, oxidative stress and apoptosis in TSCI rats, as well as the potential pathways associated.

\section{Materials and methods}

Animals. Adult male Sprague-Dawley rats (6-10 weeks; weight, 230-300 g; $n=40$ ) were obtained from the Animal Center of 
the General Hospital of Jinan Military Area Command (Jinan, China), and were housed at $23-25^{\circ} \mathrm{C}$ under a $12 \mathrm{~h}$ light/dark cycle (relative humidity 40-60\%) and fed a standard laboratory diet and water ad libitum. The present study was approved by the Scientific Review Committee and the Institutional Review board of the General Hospital of Jinan Military Area Command.

Drugs and chemicals. Methylprednisolone sodium succinate (MPSS) was supplied by Nanfang Hospital of Guangzhou in China. Superoxide dismutase (SOD; A001-3), malondialdehyde (MDA, A003-1), nuclear factor (NF)- $\kappa$ B subunit p65 (H202), tumor necrosis factor- $\alpha$ (TNF- $\alpha$, R019) and caspase-3/9 ELISA kits (catalog nos. G015 and G018) were obtained from Nanjing Jiancheng Bioengineering Institute (Nanjing, China).

TSCI model rats. The SCI model was established as previously described (7). Briefly, rats were anaesthetized by intraperitoneal (i.p.) injection of pentobarbital $(30 \mathrm{mg} / \mathrm{kg}$, Sinopharm Chemical Reagent Co., Ltd., Shanghai, China). Following anesthesia, the rat skin was shaved carefully, opened and cleaned using betadine solution. In the thoracic region, a $20 \mathrm{~mm}$ midline incision was made that exposed the vertebral column. At the tenth thoracic vertebra (T10), alaminectomy was carried out, which exposed the dorsal cord surface and left the dura intact. SCI was induced by dropping a $10 \mathrm{~g}$ rod from a height of $5.0 \mathrm{~cm}$ onto the T10 level of the spinal cord.

Experimental design. Animals in each experiment were randomly divided into 4groups $(n=10)$ : i) The control group, in which the surgical area was exposed without SCI induction and the rats received physiological saline $(0.1 \mathrm{ml} / 100 \mathrm{~g}$, i.p.); ii) the SCI group, in which SCI was induced and the rats received physiological saline $(0.1 \mathrm{ml} / 100 \mathrm{~g}$, i.p.); iii) the MPSS group, in which SCI was induced and MPSS (100 mg/kg, i.p.) was administered; and iv) the schisandrin B group, in which SCI was induced and the rats were orally administered schisandrin B (50 mg/kg/day) for 5 days.

Behavioral examination. Following schisandrin B treatment for 5 days, the rats $(n=6)$ were executed to analyze motor behavior analysis, which was performed using the Basso, Beattie and Bresnahan (BBB) locomotor scale method. BBB scores range between 0 (no observable hind-limb movements) and 21 (normal gait).

Inclined plate test. Following schisandrin B treatment for 5 days, rats $(n=6)$ were examined by the inclined plate test using a 6-mm-thick rubber pad. Rats were placed with their body axis perpendicular with orientation to the plate to evaluate the maximum vertical axis of the inclined plate. The incline angle was gradually increased, with the rats held on the inclined plate for $5 \mathrm{sec}$ and the maximum angle was recorded, with the procedure repeated three times.

Spinal cord water content. Following schisandrin B treatment for 5 days, rats $(n=6)$ were sacrificed and spinal cord samples were collected and weighed ('wet weight') and dried at $120^{\circ} \mathrm{C}$ for 24-48 h. Dry spinal cord samples were then weighed ('dry weight') and the results were recorded. The spinal cord water content was assessed by the following equation: [(wet weight-dry weight)/wet weight] x100\% (8).

Histopathological study. Spinal cord samples were fixed in $10 \%$ neutral buffered formalin for $72 \mathrm{~h}$. The tissue samples were dehydrated with gradient ethanol, soaked with xylene + ethanol for transparency and embedded in paraffin, divided into 5-mm slices and stained using hematoxylin-eosin sassy. SCI 0-4 scale: 0 , none or minor; 1 , modest or limited; 2 , intermediate; 3 , widespread or prominent and 4 , widespread and prominent.

Measurement of inflammation factors and oxidative stress. Following schisandrin B treatment for 5 days, rats $(n=6)$ were sacrificed and peripheral blood was collected. The blood was centrifuged at $12,000 \mathrm{x}$ g for $10 \mathrm{~min}$ at $4^{\circ} \mathrm{C}$ and the supernatant was collected and stored at $-80^{\circ} \mathrm{C}$ for further study. The expression levels of SOD, MDA, NF- $\mathrm{B}$ p 65 and TNF- $\alpha$ were measured using the corresponding ELISA kits (Nanjing Jiancheng Bioengineering Institute) following the manufacturer's protocol.

Western blot analysis of caspase-3 and phosphorylated (p)-p53 expression. Following schisandrin B treatment for 5 days, rats $(n=6)$ were sacrificed and spinal cord samples were collected. The spinal cord was homogenized with liquid nitrogen and radioimmunoprecipitation lysis buffer (Beyotime Institute of Biotechnology, Haimen, China). The homogenate was centrifuged at $12,000 \mathrm{x} \mathrm{g}$ for $10 \mathrm{~min}$ at $4^{\circ} \mathrm{C}$ and analyzed using a bicinchoninic acid assay kit (Beyotime Institute of Biotechnology). An equal quantity $(50 \mu \mathrm{g})$ of protein was separated by $8-10 \%$ SDS-PAGE, then transferred onto nitrocellulose filter membranes. Membranes were blocked with 5\% skim milk powder in TBST for $1 \mathrm{~h}$ at $37^{\circ} \mathrm{C}$ and detected using the following primary antibodies: Mouse anti-caspase-3 (catalog no. sc-22139; dilution, 1:300; Santa Cruz Biotechnology, Inc., Dallas, TX, USA); p-p53 expression (catalog no. sc-12904-R; dilution, 1:1,000; Santa Cruz Biotechnology, Inc.); or mouse anti- $\beta$-actin (catalog no. BB-2116-1; dilution, 1:5,000; BestBio Inc., Shanghai, China) at $4^{\circ} \mathrm{C}$ overnight. Proteins of interest were detected with horseradish peroxidase-conjugated goat anti-mouse secondary antibody (catalog no. sc-2005; dilution, 1:5,000; Santa Cruz Biotechnology, Inc.) Proteins were observed using an enhanced chemiluminescence detection ECL kit (BestBio Inc.) and quantified using imaging software (BioSens Digital Imaging 5; Shanghai Bio-Tech Inc., Shanghai, China).

Statistical analysis. The data are expressed as the mean \pm standard error of the mean. Statistical analysis was performed using two-way analysis of variance followed by Dunnett's test and the SPSS 17.0 software package (SPSS, Inc., Chicago, IL, USA) was used. $\mathrm{P}<0.05$ was considered to indicate a statistically significant difference.

\section{Results}

Effect of schisandrin B on histology following SCI induction. The chemical structure of schisandrin B is depicted in Fig. 1. The histological SCI scores were greater compared with 
control group (Fig. 2). Treatment with MPSS or schisandrin B inhibited the generation of SCI histology in SCI rats (Fig. 2).

Effect of schisandrin B on behavioral examination following $S C I$. Statistical analysis demonstrated a significant decrease in the BBB score at day 1 in the SCI, MPSS and schisandrin $\mathrm{B}$ groups compared with the control group (Fig. 3). At 5 days post SCI induction, the BBB score was significantly lower in the SCI group than the control group, and was significantly higher in the MPSS and schisandrin B groups compared with the SCI group (Fig. 3). No significant inter-group difference was identified between the schisandrin B and MPSS groups for the BBB score in SCI rats (Fig. 3).

Effect of schisandrin $B$ on the maximum angle of inclined plate test following SCI. To evaluate the protective effect of schisandrin B on SCI, the maximum angle of the inclined plate test was observed 5 days post-SCI induction. The maximum incline angle for SCI rats was significantly lower than that of control group (Fig. 4). Schisandrin B treatment exhibited a protective effect against SCI, as the maximum angle of the inclined plate was significantly higher compared with the untreated SCI group (Fig. 4). The effect of schisandrin B on SCI was similar to that of MPSS-treated SCI rats, which also exhibited significantly incline angles compared with the SCI group (Fig. 4).

Effect of schisandrin B on spinal cord water content following SCI. As shown in Fig. 5, the spinal cord water content of SCI rats was significantly increased compared with the control group. The results revealed no significant difference in the post-SCI spinal cord water content between the schisandrin Band MPSS-treated groups; however, schisandrin B and MPSS significantly lowered spinal cord water content following SCI compared with the untreated SCI group (Fig. 5).

Effect of schisandrin B on NF- $\kappa B$ p 65 and TNF- $\alpha$ expression following SCI. To evaluate the underlying mechanisms of schisandrin B in SCI, the levels of NF- $\kappa$ B p65 and TNF- $\alpha$ expression were measured 5 days following SCI induction. SCI induced significantly higher NF- $\mathrm{NB}$ p65 and TNF- $\alpha$ expression compared with the control group (Fig. 6). By contrast, treatment with schisandrin B or MPSS significantly inhibited the SCI-induced expression of NF- $\kappa \mathrm{B}$ p65 and TNF- $\alpha$ in SCI rats (Fig. 6); no significant difference was observed between the MPSS-treated and schisandrin B-treated groups (Fig. 6).

Effect of schisandrin B on oxidative stress following SCI. MDA and SOD expression levels in spinal cord tissue were also measured to fully investigate the mechanisms involved in the effect of schisandrin B in SCI. As shown in Fig. 7, MDA expression was significantly increased and SOD expression was significantly decreased in the SCI group compared with the control group. However, treatment with schisandrin B significantly lowered MDA expression levels and significantly increased SOD expression compared with the untreated SCI group (Fig. 7). The results demonstrated that there were no significant differences in MDA and SOD activity between the schisandrin B-and MPSS-treated groups at 5 days following the SCI surgery.

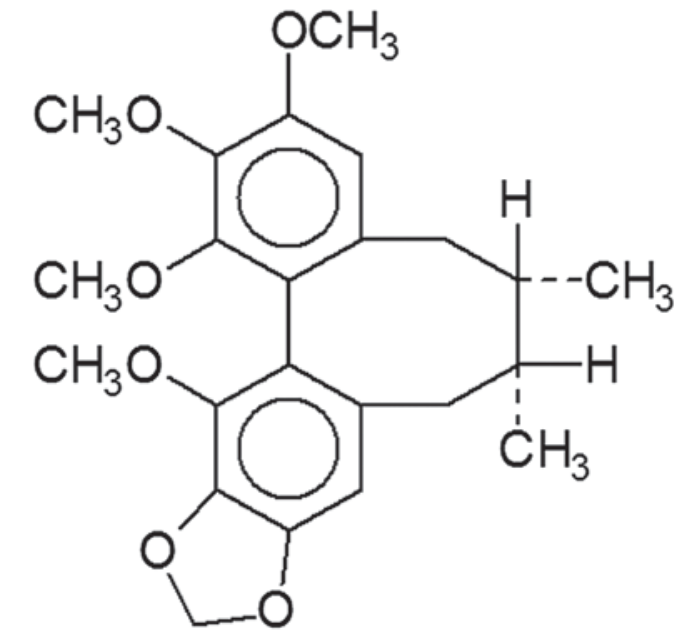

Figure 1. Chemical structure of schisandrin B.

Effect of schisandrin B on apoptosis following SCI. The effect of schisandrin B on apoptosis was investigated by measuringcaspase-3 and p-p53 protein expression. There was a significant increase in caspase-3 and p-p53 protein expression following SCI compared with the control group (Fig. 8). Schisandrin B treatment significantly alleviated this increase in caspase-3 and p-p53 protein expressions in SCI rats, compared with untreated SCI group (Fig. 8). In addition, no significant difference was identified in caspase-3 and p-p53 protein expression between the schisandrin B-and the MPSS-treated groups (Fig. 8).

\section{Discussion}

TSCI is characterized by spinal cord damage caused by a number of different factors, such as trauma and infection, which can lead to symptoms of paralysis and serious debilitating diseases. Rapid developments in transportation, engineering construction and the sports entertainment industry have led to arise in the incidence of TSCI (3). It has been reported that $>3$ million people are affected by TSCI worldwide. The pathological process of acute secondary spinal cord damage is complex owing to the release of a number of factors, including excitatory amino acids, oxidizing metabolites and inflammatory cells, which lead to irreversible damage as well as movement, sensory and autonomic nerve dysfunction $(4,9)$. In the present study, schisandrin B treatment improved the $\mathrm{BBB}$ score and the maximum angle of the inclined plate test, and reduced the spinal cord water content in TSCI rats. These results indicate that schisandrin B possesses beneficial properties against TSCI.

TSCI is one of the leading causes of disability, and its pathological process includes primary and secondary injuries. Secondary injury initially stimulates a large number of inflammatory cells, which produce a strong inflammatory reaction and eventually glial cell death (10). Inflammation factors is a major neurotransmitter regulating immune response, it is the Bridges of our immune cells and other cells (10). NF- $\kappa \mathrm{B}$ p65 and TNF- $\alpha$ are secreted by activated microglia and macrophages, neurons and glial cells during the early stages of TSCI (11). As neurotransmitters for intercellular signal transduction, $\mathrm{NF}-\kappa \mathrm{B}$ p 65 and $\mathrm{TNF}-\alpha$ regulate the central 


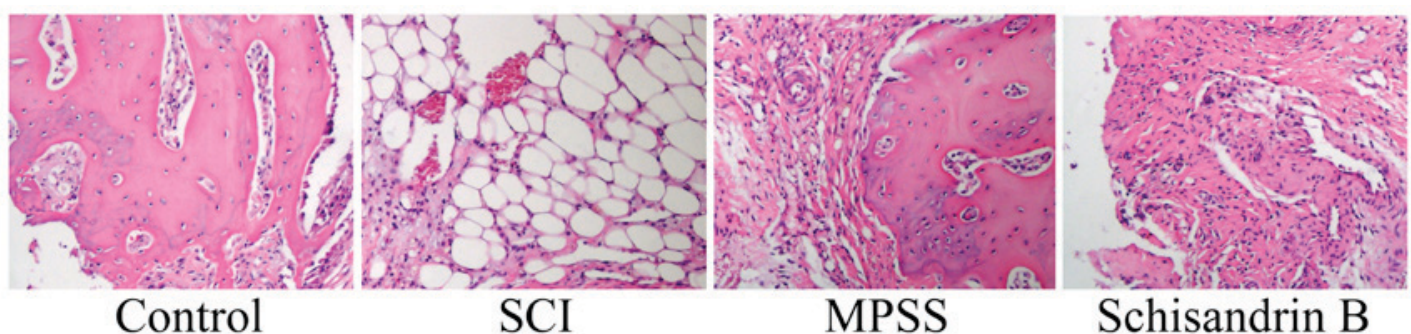

Figure 2. Effect of schisandrin B on histological examination following SCI. Representative images of spinal cord tissue in the control, SCI, MPSS-treatment and schisandrin B-treatment groups. MPSS, methylprednisolone sodium succinate; SCI, spinal cord injury (magnification, x40 times).

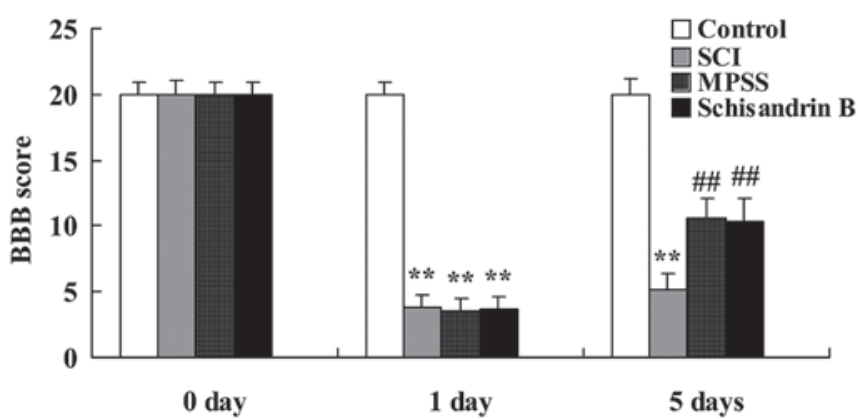

Figure 3. Effect of schisandrin B on behavioral examination following SCI. Post-SCI locomotor behavior was assessed using the BBB locomotor scale method. BBB scores range between 0 (no observable hind-limb movements) and 21 (normal gait). Data are presented as the mean \pm standard error of the mean. ${ }^{* *} \mathrm{P}<0.01$ vs. control group; ${ }^{\# \#} \mathrm{P}<0.01$ vs. untreated SCI group. $\mathrm{BBB}$ Basso, Beattie and Bresnahan; MPSS, methylprednisolone sodium succinate; $\mathrm{SCI}$, spinal cord injury.

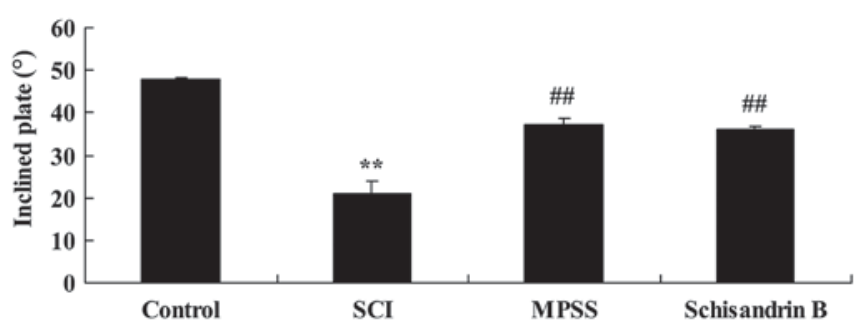

Figure 4. Effect of schisandrin B on the maximum angle of inclined plate test following SCI. To evaluate the protective effect of schisandrin B on SCI, the maximum angle of the inclined plate test was observed 5 days post-SCI. Data are presented as the mean \pm standard error of the mean. ${ }^{* *} \mathrm{P}<0.01$ vs. control group; ${ }^{\# \#} \mathrm{P}<0.01$ vs. untreated SCI group. MPSS, methylprednisolone sodium succinate; SCI, spinal cord injury.

nervous system, inflammation and autoimmune reactions (12). TNF- $\alpha$ promotes polymorphonuclear leukocyte and macrophage infiltration from the blood to the area of inflammation, and also promotes the activation of astrocytes, resulting in the formation of a glial scar that can induce a permanent loss of neurological function (13). The present study demonstrated that schisandrin B effectively inhibited the SCI-induced activation of NF- $\mathrm{kB}$ p 65 and TNF- $\alpha$ in TSCI rats. Giridharan et al demonstrated that schisandrin B attenuates cisplatin-induced oxidative stress and inflammation in mice (14), and Thandavarayan et al revealed that schisandrin B may be preventive against doxorubicin-induced cardiac dysfunction through the inhibition of oxidative stress and inflammation (6).

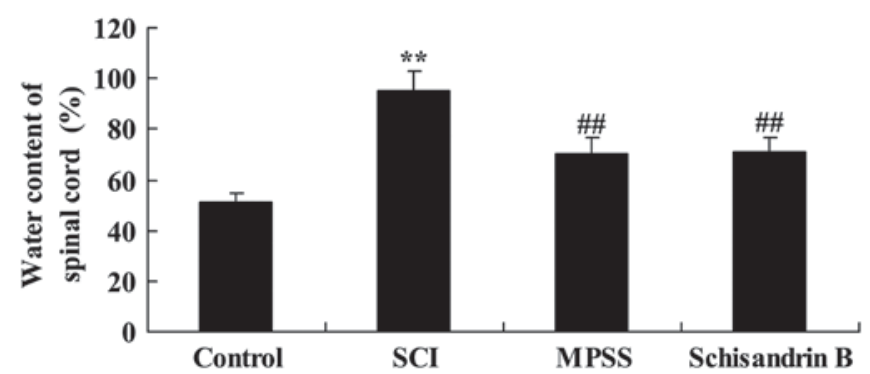

Figure 5. Effect of schisandrin B on spinal cord water content following SCI. Spinal cord water content was assessed by the following equation: [(wet weight-dry weight)/wet weight] $\times 100 \%$. Data are presented as the mean \pm standard error of the mean. ${ }^{* *} \mathrm{P}<0.01$ vs. control group; ${ }^{\# \#} \mathrm{P}<0.01$ vs. untreated SCI group. MPSS, methylprednisolone sodium succinate; SCI, spinal cord injury.

Oxidative stress is a basic protective mechanism to regulate normal activities in the body, including cell signal transduction, proliferation and apoptosis (15). Oxidative stress is characterized by the increased production of reactive oxygen species (ROS) and reactive nitrogen species (RNS). ROS and RNS can be generated in a number of ways, including chemical and drug metabolism, cell respiration (12). However, an excessive level of free radicals inhibits the body's ability to remove oxides, which can lead to a high level of oxidative damage (16). The spinal cord contains a large number of polyunsaturated fatty acids, has limited neuronal regeneration ability and an active oxidative metabolism; however, it has a low antioxidant capacity, therefore reactive oxygen metabolites accumulate and antioxidants are excessively reduced in this tissue (16). These characteristics make neurons and glial cells particularly susceptible to the influence of harmful stimulation and oxidative stress (17). The present study revealed that schisandrin B treatment effectively inhibited the SCI-induced expression of MDA in TSCI rats. Schisandrin B treatment also improved the SCI-induced reduction in SOC expression in TSCI rats. Chen $e t a l$ reported that schisandrin B protects against cerebral ischemia/reperfusion injury through the antioxidant status in rats (5).

Apoptosis is different from necrocytosis in that it is a process of programmed cell death that is regulated by a variety of signaling pathways and results in characteristic morphological changes and DNA fragmentation (18). Caspases serve a key role in apoptotic events, adjust cell death, and the appearance and function of Caspase apoptotic features are closely associated (19). They cut off the target cells from the 

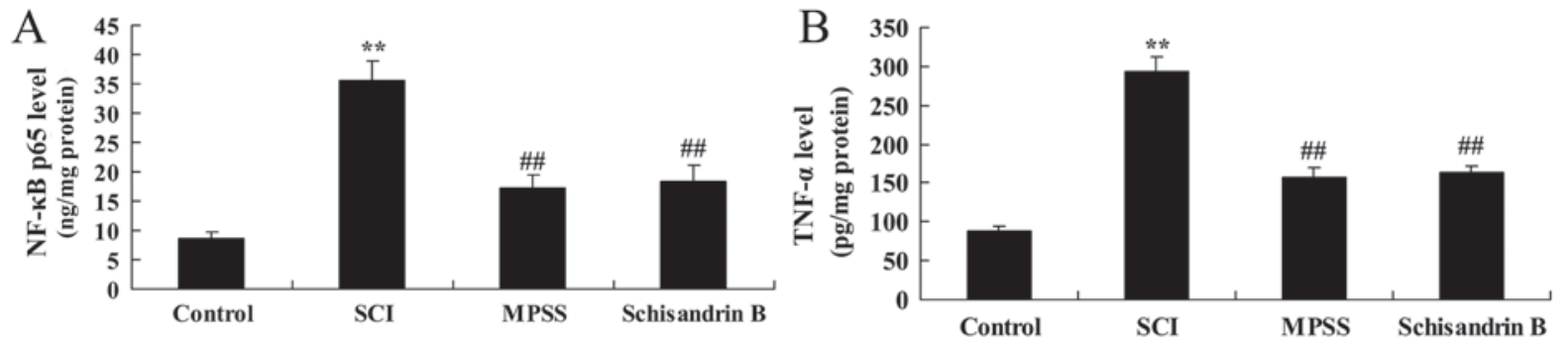

Figure 6. Effect of schisandrin B on NF- $\kappa$ B subunit p65 and TNF- $\alpha$ expression following SCI. The levels of (A) NF- $\mathrm{B}$ p 65 and (B) TNF- $\alpha$ expression following the induction of SCI. Data are presented as the mean \pm standard error of the mean. ${ }^{* *} \mathrm{P}<0.01$ vs. control group; ${ }^{\# \#} \mathrm{P}<0.01$ vs. untreated $\mathrm{SCI}$ group. MPSS, methylprednisolone sodium succinate; NF- $\mathrm{kB}$, nuclear factor-kB; SCI, spinal cord injury; TNF- $\alpha$, tumor necrosis factor $\alpha$.
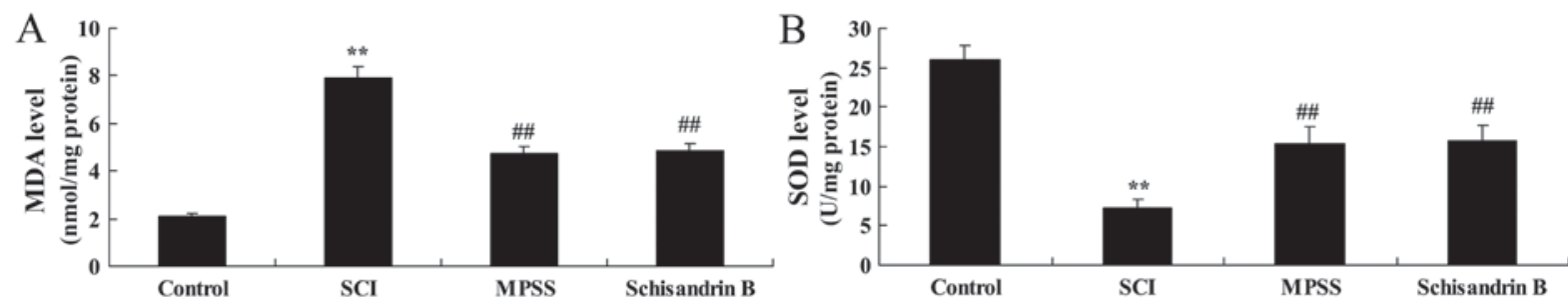

Figure 7. Effect of schisandrin B on oxidative stress following SCI. The levels of (A) MDA and (B) SOD expression following the induction of SCI. Data are presented as the mean \pm standard error mean. ${ }^{* *} \mathrm{P}<0.01$ vs. control group; ${ }^{\# \#} \mathrm{P}<0.01$ vs. untreated SCI group. MDA, malondialdehyde; MPSS, methylprednisolone sodium succinate; SCI, spinal cord injury; SOD, superoxide dismutase.
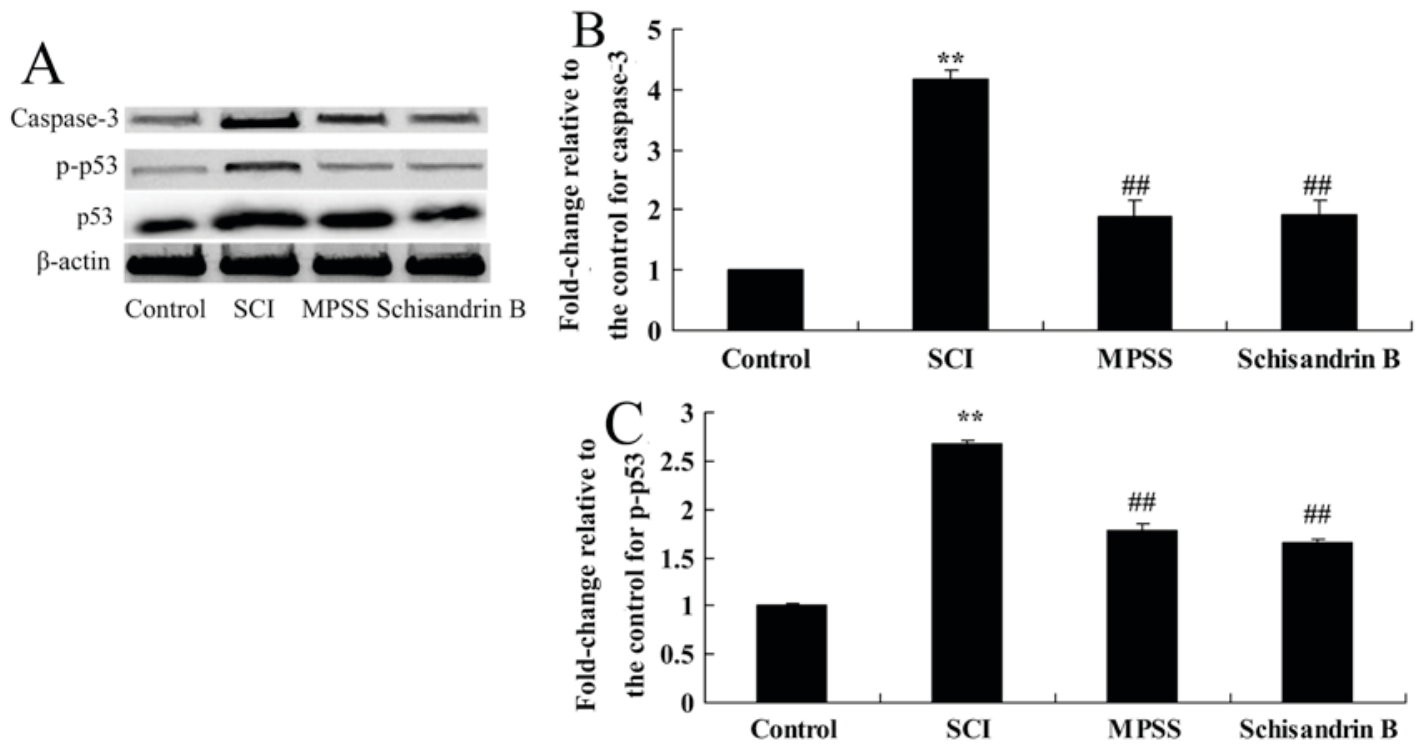

Figure 8. Effect of schisandrin B on caspase-3 and p-p53 protein expression following SCI. The effect of schisandrin B on caspase-3 and p-p53 protein expression following the induction of SCI was evaluated by (A) western blotting and (B and C) densitometric analysis. Protein expression was normalized to $\beta$-actin. Data are presented as the mean \pm standard error of the mean. ${ }^{* *} \mathrm{P}<0.01 \mathrm{vs.}$ control group; ${ }^{* \#} \mathrm{P}<0.01$ vs. untreated SCI group. MPSS, methylprednisolone sodium succinate; SCI, spinal cord injury.

surrounding cells, reorganize the cytoskeleton, lower the level of DNA replication and cell repair abilities, damage the DNA and nuclear structure, attract phagocytes and disintegration of the cell formation apoptotic body (20). Previous studies have confirmed that promotion of caspase activity in TSCI model rats increases neural cell apoptosis $(19,20)$. The present study demonstrated that schisandrin B treatment significantly reduced the SCI-induced increase in caspase- 3 protein expression in SCI rats. Wang et al revealed that schisandrin
B protects against amyloid $\beta$ (1-42)-induced neurotoxicity in cortical neurons by decreasing caspase- 9 and caspase- 3 activities (21). Chiu et al demonstrated that schisandrin B protects against hypoxia/reoxygenation-induced apoptosis through the suppression of caspase-3 protein expression in mouse AML12 hepatocytes (22).

p53 is important in TSCI and regulates spinal cord apoptosis to accelerate the death of oligodendrocytes, microglia and astrocytes (23). p53 may additionally have a role in the 
inhibition of DNA repair (24). Future research may investigate how to assess the development of this regulation, the damage to DNA repair and how to improve the survival rate of neurons and glial cells $(24,25)$. In the present study, schisandrin B significantly reduced the activation of $\mathrm{p}$-p53 protein expression in SCI rats.

The present study demonstrated that schisandrin B attenuated the inflammatory response, oxidative stress and apoptosis in TSCI model rats by inhibiting the p53 signaling pathway. Further clinical studies are required to determine whether schisandrin B would be effective in anti-inflammatory, antioxidative and anti-apoptotic therapies for TSCI.

\section{Acknowledgements}

The present study was partly supported by The Youth Innovation Fund of Inner Mongolia Medical University (grant no. YKD2014QNCX021).

\section{References}

1. Cristante AF, Barros Filho TE, Marcon RM, Letaif OB and Rocha ID: Therapeutic approaches for spinal cord injury. Clinics (Sao Paulo) 67: 1219-1224, 2012.

2. Paula AA, Nicolau RA, Lima Mde O, Salgado MA and Cogo JC: 'Low-intensity laser therapy effect on the recovery of traumatic spinal cord injury'. Lasers Med Sci 29: 1849-1859, 2014.

3. Chun S and Lee Y: 'I am just thankful': The experience of gratitude following traumatic spinal cord injury. Disabil Rehabil 35: 11-19, 2013.

4. Rahimi-Movaghar V, Niakan A, Haghnegahdar A, Shahlaee A, Saadat $\mathrm{S}$ and Barzideh E: Early versus late surgical decompression for traumatic thoracic/thoracolumbar (T1-L1) spinal cord injured patients. Primary results of a randomized controlled trial at one year follow-up. Neurosciences (Riyadh) 19: 183-191, 2014

5. Chen N, Chiu PY and Ko KM: Schisandrin B enhances cerebral mitochondrial antioxidant status and structural integrity, and protects against cerebral ischemia/reperfusion injury in rats. Biol Pharm Bull 31: 1387-1391, 2008.

6. Thandavarayan RA, Giridharan VV, Arumugam S, Arumugam S, Suzuki K, Ko KM, Krishnamurthy P, Watanabe K and Konishi T: Schisandrin B prevents doxorubicin induced cardiac dysfunction by modulation of DNA damage, oxidative stress and inflammation through inhibition of MAPK/p53 signaling. PLoS One 10: e0119214, 2015.

7. Gruner JA: A monitored contusion model of spinal cord injury in the rat. J Neurotrauma 9: 123-128, 1992.

8. Guo J, Li Y, He Z, Zhang B, Li Y, Hu J, Han M, Xu Y, Li Y, $\mathrm{Gu} \mathrm{J}$, et al: Targeting endothelin receptors $\mathrm{A}$ and $\mathrm{B}$ attenuates the inflammatory response and improves locomotor function following spinal cord injury in mice. Int J Mol Med 34: 74-82, 2014.

9. van Middendorp JJ, Barbagallo G, Schuetz M and Hosman AJ: Design and rationale of a prospective, observational European Multicenter Study on the efficacy of acute surgical decompression after traumatic spinal cord injury: The SCI-POEM study. Spinal Cord 50: 686-694, 2012.

10. Zhang D, Yue Y, Jiang S, Li A, Guo A, Wu X, Xia X, Cheng H, Zhang J, Tao T and Gu X: GART expression in rat spinal cord after injury and its role in inflammation. Brain Res 1564: 41-51, 2014.
11. Ni H, Jin W, Zhu T, Wang J, Yuan B, Jiang J, Liang W and Ma Z: Curcumin modulates TLR4/NF- $\kappa$ B inflammatory signaling pathway following traumatic spinal cord injury in rats. J Spinal Cord Med 38: 199-206, 2015.

12. Tan J, Zhang F, Liang F, Wang Y, Li Z, Yang J and Liu X: Protective effects of hyperbaric oxygen treatment against spinal cord injury in rats via toll-like receptor $2 /$ nuclear factor- $\mathrm{\kappa} \mathrm{B}$ signaling. Int J Clin Exp Pathol 7: 1911-1919, 2014

13. Genovese T, Mazzon E, Crisafulli C, Di Paola R, Muià C, Bramanti P and Cuzzocrea S: Immunomodulatory effects of etanercept in an experimental model of spinal cord injury. J Pharmacol Exp Ther 316: 1006-1016, 2006.

14. Giridharan VV, Thandavarayan RA, Bhilwade HN, Ko KM, Watanabe K and Konishi T: Schisandrin B, attenuates cisplatin-induced oxidative stress, genotoxicity and neurotoxicity through modulating NF- $\kappa \mathrm{B}$ pathway in mice. Free Radic Res 46: 50-60, 2012.

15. Ordonez FJ, Rosety MA, Camacho A, Rosety I, Diaz AJ, Fornieles G, Bernardi M and Rosety-Rodriguez M: Arm-cranking exercise reduced oxidative damage in adults with chronic spinal cord injury. Arch Phys Med Rehabil 94: 2336-2341, 2013.

16. Kurtoglu T, Basoglu H, Ozkisacik EA, Cetin NK, Tataroglu C, Yenisey C and Discigil B: Effects of cilostazol on oxidative stress, systemic cytokine release, and spinal cord injury in a rat model of transient aortic occlusion. Ann Vasc Surg 28: 479-488, 2014.

17. Juang CL, Yang FS, Hsieh MS, Tseng HY, Chen SC and Wen HC: Investigation of anti-oxidative stress in vitro and water apparent diffusion coefficient in MRI on rat after spinal cord injury in vivo with Tithonia diversifolia ethanolic extracts treatment. BMC Complement Altern Med 14: 447, 2014.

18. Zhang H, Wu F, Kong X, Yang J, Chen H, Deng L, Cheng Y, Ye L, Zhu S, Zhang X, et al: Nerve growth factor improves functional recovery by inhibiting endoplasmic reticulum stress-induced neuronal apoptosis in rats with spinal cord injury. J Transl Med 12: 130, 2014

19. Ersahin M, Çevik Ö, Akakın D, Şener A, Özbay L, Yegen BC and Sener G: Montelukast inhibits caspase-3 activity and ameliorates oxidative damage in the spinal cord and urinary bladder of rats with spinal cord injury. Prostaglandins Other Lipid Mediat 99: 131-139, 2012.

20. Springer JE, Azbill RD and Knapp PE: Activation of the caspase-3 apoptotic cascade in traumatic spinal cord injury. Nat Med 5: 943-946, 1999

21. Wang B and Wang XM: Schisandrin B protects rat cortical neurons against Abeta1-42-induced neurotoxicity. Pharmazie 64: 450-454, 2009

22. Chiu PY, Luk KF, Leung HY, Ng KM and Ko KM: Schisandrin B stereoisomers protect against hypoxia/reoxygenation-induced apoptosis and associated changes in the $\mathrm{Ca}(2+)$-induced mitochondrial permeability transition and mitochondrial membrane potential in AML12 hepatocytes. Phytother Res 23: 1592-1602, 2009.

23. Floriddia EM, Rathore KI, Tedeschi A, Quadrato G, Wuttke A, Lueckmann JM, Kigerl KA, Popovich PG and Di Giovanni S: p53 Regulates the neuronal intrinsic and extrinsic responses affecting the recovery of motor function following spinal cord injury. J Neurosci 32: 13956-13970, 2012.

24. Chopp M, Chan PH, Hsu CY, Cheung ME and Jacobs TP: DNA damage and repair in central nervous system injury: National Institute of Neurological Disorders and Stroke Workshop Summary. Stroke 27: 363-369, 1996.

25. Cai Y, Li J, Yang S, Li P, Zhang X and Liu H: CIBZ, a novel BTB domain-containing protein, is involved in mouse spinal cord injury via mitochondrial pathway independent of p53 gene. PLoS One 7: e33156, 2012. 